Trabajos y Comunicaciones, 2da. Época, No 47, e054, enero-junio 2018. ISSN 2346-8971

Universidad Nacional de La Plata.

Facultad de Humanidades y Ciencias de la Educación.

Departamento de Historia

\title{
Nacionalismo, insurreccion armada, antisemitismo: florescencias de derecha en el ecosistema posperonista
}

\author{
Nationalism, armed insurrection, anti-semitism: blossomings of the \\ right in the postperonist ecosystem
}

\section{Carolina Cerrano * Héctor Ghiretti **}

* Universidad de Montevideo/ Agencia Nacional de Investigación e Innovación, Uruguay ccerrano@um.edu.uy

** Instituto de Ciencias Humanas, Sociales y Ambientales-CONICET / Universidad Nacional de Cuyo, Argentina | hector.ghiretti@gmail.com

\section{PALABRAS CLAVE}

Derecha

Argentina

Post-peronismo

KEYWORDS

Right

Argentine

Postperonismo

\section{RESUMEN}

La aplicación del tándem categorial izquierda-derecha al universo político posee como tal una serie de demandas y condiciones que frecuentemente se pasan por alto. Estas vienen dadas por el analogado principal de estos conceptos, que es el de las distinciones corporales-espaciales. Pero además, es preciso advertir que en la dinámica que lleva a los conceptos políticos desde la autoiluminación (Voegelin) propia de los actores políticos a su formalización apta para el análisis teórico, izquierda y derecha siguen itinerarios muy diversos. Eso explica la usual resistencia de los agentes políticos de derecha a ser clasificados según esa categoría y también la inestabilidad/indefinición propia de ese término, sobre todo si se lo compara con su opuesto, la izquierda. En nuestro país, izquierda y derecha como identidades políticas poseen un surgimiento tardío, delimitado a procesos y actores políticos sustancialmente diversos a aquellos que en los países del continente europeo les dieron luz. Una particularidad local es que no se aparecen como clasificaciones propias de las facciones internas de órganos deliberativos sino por fuera de ellos. Nuestra hipótesis es que la más cercana configuración de un modelo de izquierda y derecha en la Argentina tuvo lugar como diferenciación de las diversas líneas internas del peronismo, con posterioridad al cierre de su período clásico (1946-1955)

\section{ABSTRACT}

The application of the left-right categorical tandem to the political universe possesses as such, a number of demands and conditions that are frequently overlooked. These demands and conditions are a product of the main analogy of these concepts: the corporal spatial distinctions. But in addition, it is important to note that in the dynamics of the political concepts from the self-understanding (Voegelin) of the political actors to their formalization -suitable for the theoretical analysis- the left and the right walk through very different roads. This situation explains the usual resistance of the political agents of the right to be classified under that category, and also the instability/uncertainty of that label, particularly when compared with its opponent, the left. In our country, left and right as political identities possess a late emergence, enclosed to processes and political actors substantially diverse to those that in the European continent gave birth to them. A local particularity is that they do not appear as categories of the internal features of deliberative organs, but apart from them. Our hypothesis is that in Argentina, the closest configuration of a model of left and right took place as a differentiation of the diverse internal lines of the Peronism, after the end of its classical period (1946-1955). 


\section{Las exigencias epistemológicas de una categoría dual $\underline{1}$}

El propósito de analizar los actores del escenario político argentino a partir de las categorías de izquierda y derecha responde a una decisión epistemológica que requiere una sólida fundamentación, por dos motivos concurrentes.

En primer lugar, no son categorías que corresponden a objetos “reales”, es decir, identidades políticas presentes en dicho escenario, ni tampoco racionalizaciones historiográficas de fenómenos bien definidos, con contornos bien delimitados. No es lo mismo estudiar el peronismo, la Unión Cívica Radical o el gobierno de Roca que la Generación del 80 por un lado, o el conservadurismo, los procesos de modernización o las diversas encarnaciones del populismo por el otro. Por su condición difusa y su marginal presencia en el espectro de las identidades políticas autoconscientes, izquierda y derecha pertenecen, al menos en el contexto argentino y latinoamericano, a un universo de racionalizaciones de tercer grado.

En segundo lugar, poseen una lógica característica que se deriva de su originaria índole espacial. La distinción entre izquierda-derecha supone un continuum espacial de disposición horizontal. La condición de posibilidad de aplicación de la distinción es que todos los objetos relacionados (organizaciones, líderes o intelectuales, ideas, ideologías) puedan ser alineados sobre el continuo mencionado según criterios de moderación o radicalización, no aceptando presencias disruptivas. Se trata de un esquema excluyente, incompatible con otras distinciones, espaciales o no.

Asimismo, cualquiera sea la definición que se les dé, es necesario que los términos de la clasificación tengan entre sí una relación de simetría de oposición de tipo especular o reflectivo. Siempre dentro de lo que permite la metáfora, los elementos ideológicos o actitudinales que existen a un lado y el otro deben oponerse entre sí. $\underline{2}$

Si no hay oposición entre izquierda y derecha las categorías son inaplicables. Esto implica la necesidad de "reducir" o "aplanar" a tales criterios todo fenómeno u objeto político contemporáneo, aún si presentan problemas serios en términos de identificación según este eje. El esquema de clasificación y racionalización derechaizquierda empieza a tener un valor cuestionable si elementos sustanciales del corte de la realidad que se somete a análisis no pueden ser reducidos a esta lógica espacial.

Si el peronismo o el radicalismo pierden características diferenciales sustantivas al ser ajustadas a las distinciones entre izquierda y derecha, entonces estas no pueden constituirse en categorías primarias de identificación y racionalización política. Esto no es una particularidad argentina: la mayoría de las culturas políticas de los países occidentales no se ajustan bien a la distinción bilateral.

Si la izquierda se convierte en una identidad "encapsulada”, sin continuidad hacia las zonas medias o moderadas del espectro ¿tiene más sentido de hablar de "izquierda" que referirse a esas identidades como socialistas y/o anarquistas? Es sabido que en contextos de fuerte densidad ideológica las diversas variantes no solamente no admiten que exista una categoría que las incluya a todas, sino que cada una de ellas usualmente impugna con diversos argumentos a las demás. ¿No se constituirá en una categoría equívoca, quizá útil para constituir identidades marginales, pero ineficaz a los efectos de valerse de un concepto teóricamente útil, que no cumple condiciones elementales de ordenamiento espacial a lo largo de un eje horizontal y articulado por una relación de simetría especular?

Pero si esos problemas se encuentran en un extremo del espectro, en el otro son aún mayores. En el universo de los las categorías políticas existen elementos que nadie discute en tanto tales -aunque todos estén comprendidos bajo el régimen de difusividad y problematicidad en torno a su definición, como el resto de las nociones propias de las ciencias sociales- y otros que son constantemente cuestionados, es decir, en tanto principios que permiten comprender y clasificar fenómenos dentro del universo político. Este es el caso del concepto o categoría de derecha.

Como intentaremos mostrar en este breve estudio introductorio, el concepto de derecha no solamente recibe 
cuestionamientos e impugnaciones por el hecho de pertenecer al siempre problemático par conceptual izquierdaderecha, sino que aún aceptando el valor teórico de la distinción dual, la derecha está sometida a impugnaciones específicas. Únicamente reconstruyendo el itinerario de la derecha como categoría política es posible comprender sus confines de racionalización teórica y a la vez su utilidad como concepto en el universo de las identidades políticas.

En su notable Nueva ciencia de la política, Eric Voegelin (1968) explica que el científico político no encuentra un vacío en el que formula sus interpretaciones sobre el orden social y los símbolos que lo representan.

“La ciencia política atraviesa por una dificultad que tiene su origen en su misma naturaleza de ciencia del hombre en existencia histórica. El hombre no espera a que la ciencia venga a explicarle su propia vida, y cuando el teórico se acerca a la realidad social se encuentra con el terreno ya ocupado por lo que podría denominarse auto-interpretación de la sociedad. La sociedad humana no es un mero hecho, o un suceso del mundo exterior que pueda ser estudiado por un observador como si fuese un fenómeno natural” (47-48).

Esta autointerpretación se realiza en buena medida por medio de símbolos, los cuales logran el efecto de autoiluminación. "Los símbolos hacen que la estructura interna de este cosmos, las relaciones entre sus miembros y grupos de miembros, así como su existencia como conjunto, sean transparentes para ese misterio que es el existir del hombre” (Voegelin, 1968: 47-48). La sociedad, así concebida, resulta una "especie de cosmos de significación iluminado desde dentro por su propia autointerpretación” (Voegelin, 84). El autor afirma que “la ciencia política parte del cuerpo de autointerpretación de la sociedad, y avanza por medio de la clarificación crítica de esos símbolos socialmente preexistentes”.

El proceso de racionalización política tiende a producir un nuevo conjunto de símbolos, ya propio de la clarificación crítica. “La reflexión teórica sobre lo político” -prosigue Voegelin- "se produce disponiendo de dos juegos de símbolos: uno del lenguaje, propio de la autointerpretación, y otro, propio de la ciencia política.” Uno se deriva del otro. Es tarea del científico determinar si "los símbolos utilizados en la realidad política son conceptos teoréticos” (Voegelin, 49-51), ya que a menudo los símbolos no poseen dicha virtualidad y requieren de un marco teórico más amplio para poder ser racionalizados y comprendidos.

\section{Lógica y dinámica de los conceptos posrevolucionarios}

Es precisamente ese el camino de la categoría política que conocemos como izquierda. Su aparición en el universo de los conceptos políticos de la modernidad posrevolucionaria no es tan disruptiva ni tan lineal (u homogénea) como pudiera parecer. Si bien existe un consenso entre los estudiosos en torno al momento histórico en que surgió, también es cierto que la distinción izquierda-derecha es un complejo de categorías corporales-espaciales de fuerte proyección simbólica que se encuentra en casi todas las culturas y del que la conceptualización política contemporánea no es en absoluto ajena.

La asociación entre el espacio físico que ocuparon, dentro del nuevo recinto asambleario, los diversos estamentos de los antiguos États Généraux, y las ideas y doctrinas en torno a la legitimidad del poder y el orden político que cada uno de ellos sostenía, supuso una resemantización del binomio que se operó en los primeros días de la Assemblée Nationale, lo cual supuso un espectacular fenómeno de inversión valorativa en el plano simbólico. Pero esta asociación no fue evidente para los protagonistas u observadores políticos de la época, ni se adquirió plena conciencia de ella de forma inmediata. La distinción siguió las peripecias de la accidentada historia política del s. XIX francés, paralela a la presencia y la actividad intermitente de cuerpos deliberativos. Tuvo que pasar un largo siglo para que la izquierda desbordara los recintos asamblearios y se constituyera en una identidad política propiamente dicha, trascendiendo las referencias a los espacios concretos, tanto deliberativos como nacionales (Gauchet, 1992). 
La pregunta por el significado político de la izquierda y la derecha, en su acepción y empleo posrevolucionario, puede remontarse al menos a mediados del s. XIX (Duclerc y Pagnerre, 1848: 425-426). La discusión sobre tales conceptos, no obstante, tiene lugar en Francia recién en la década de 1930, en un contexto político e intelectual cuyas características empujaban decididamente a su revisión. $\underline{3}$ Tal discusión forma parte del proceso de constitución -a través de la clarificación crítica- de la izquierda y la derecha como categorías políticas, como segundo conjunto de símbolos -para usar la terminología de Voegelin- propios de la ciencia política, que se sucede al primer conjunto de símbolos, propios de la fase operativa y autointerpretativa de la política.

Dentro de ese par conceptual, curiosamente, la evolución de la derecha es diversa de la que sigue la izquierda. El concepto político de derecha no respondió a una necesidad de autointerpretación en términos estrictos, sino a la necesidad de representación de una alteridad radical, de la constitución de un adversario que se oponía al proyecto de modernización política, económica y social conocido como izquierda.

Se trató de algo que podríamos llamar una autointerpretación in alio, -es decir, la constitución de una alteridad que sirve para constituir la identidad propia, en un despliegue auténticamente dialéctico- y por esa razón, no generó una autoidentidad, tal como sucede con la izquierda, sino una identidad heteroatribuida, respecto de la cual quienes estaban comprendidos por ella no se vieron identificados ni representados.

No nos es posible entrar en el estudio sobre el despliegue de la distinción izquierda y derecha a partir de la dialéctica moderna. Nos interesa dar cuenta del fenómeno más bien desde la perspectiva de sus manifestaciones. El desafecto de los actores políticos tradicionalmente identificados con la derecha respecto de la categoría que los definía fue tempranamente advertido por los observadores de la política francesa de entreguerras. Puede verse en un célebre texto de Alain:

"La primera idea que me viene a la cabeza cuando alguien me pregunta si la distinción entre partidos de izquierda y derecha, entre hombres de izquierda y de derecha, tiene todavía sentido, es que quien me lo pregunta no es ciertamente un hombre de izquierda” (Alain, 1934: 68). 4

Esta particularidad también ha sido explicada por autores tradicionalistas franceses (Madiran, 1979). Tal característica originaria de la derecha ha sido causa de no pocos malos entendidos y confusiones. Es el caso de las tesis de Gustavo Bueno (2003) que sostiene que la derecha precede en el tiempo a la izquierda, e incluso que se puede hablar de una derecha eterna. La verdad es que, siempre y en todos los casos la constitución de una derecha es efecto o producto de la emergencia de la identidad de izquierda.

La categoría política de derecha, pues, no se desarrolla a partir de una autoidentidad, razón por la cual no hay, por así decirlo, un tránsito de la subjetividad (como autoidentidad política) a la objetivación (como categoría de análisis de la ciencia política) tal como sucede con la izquierda. Lo cual permite ciertas libertades y variaciones en lo que hace a su definición y su aplicación como categoría.

En primer lugar, no hay nadie que plantee un conflicto entre lo que es la derecha como identidad propia y lo que la crítica entiende por derecha. No hay corrección hermenéutica posible desde una derecha como autoidentidad. Esto evita los engorrosos debates o disputas teóricas y/o políticas en torno a qué es (o más bien qué está a) la izquierda, las cuales se dan usualmente según dos líneas argumentales contrapuestas: las encarnaciones posibles -históricas, circunstanciadas, imperfectas- de la identidad de izquierda (la izquierda real) y la izquierda como identidad ideal o perfecta (la izquierda ideal o eterna).

En segundo lugar, la derecha en tanto que concepto constituido como la alteridad opuesta a la izquierda posee una plasticidad que no se limita a lo táctico sino que posee un alcance estratégico. Así, derecha no es solamente (y tampoco esencialmente) aquella facción política que acepta y asume el esquema bipolar, es decir se define como una antiizquierda, sino que es todo aquello que se opone, es obstáculo, bloquea o combate a la izquierda, o simplemente es el objetivo a destruir o reformar para que la izquierda pueda cumplir su objetivo, es decir, una no- 
izquierda. En este sentido, toda expresión o condensación del orden que debe demolerse/transformarse, instituciones, tradiciones, grupos sociales, corporaciones, sistemas completos (el Antiguo Régimen) y hasta cosmovisiones también se convierten en la derecha.

Estas determinaciones son siempre unidireccionales: la condición de posibilidad para que sea posible distinguir una derecha es que exista una identidad que se recorte del universo político en términos -si no explícitos, al menos implícitos- de izquierda. Tal determinación no funciona a la inversa: la distinción derecha-izquierda nunca procede por definición primaria de una derecha. La derecha siempre es una categoría por defecto, derivada: más todavía en el caso de que esta finalmente constituya una identidad, es decir, trascienda el carácter de adversario político y se autoasuma como identidad.

La dinámica de las distinciones bipolares siempre inicia por izquierda. Esto se da tanto en el plano de las identidades como en el de las categorías de análisis. Basta que, a partir de un cuestionamiento radical al orden existente, una facción se proponga llevar a cabo un proyecto opositor/alternativo de transformación en un sentido emancipador o igualitario, para que todo el universo político pueda ser desplegado dentro de un esquema clasificatorio simbólico horizontal.

Esta cuestión conlleva dificultades propias: la desproporción de los elementos enfrentados -el orden vigente, con toda su dimensión, su complejidad y su poder, contra quienes se enfrentan a él- puede resultar un poderoso aliciente para la constitución de la autoidentidad de izquierda, pero no lo es en absoluto para la heteroidentidad de derecha, y posee una relativa validez como categoría política de análisis a partir de este última, aún cuando se muestre extremadamente flexible a los efectos de incluir en esa última una gran diversidad de elementos.

Pero entonces ¿constituyen nociones útiles para racionalizar los elementos del universo político? ¿Cómo conciliar epistemológicamente el complejo de instituciones, creencias, símbolos, leyes, tradiciones, grupos sociales que la izquierda pretende abolir o combatir (una derecha en sentido amplio, oceánico: una no-izquierda) y los sectores que advierten la amenaza o el desafío planteado por la izquierda y aceptan la confrontación (una derecha en sentido estricto, independientemente del hecho de que acepten el término de definición: una antiizquierda)?

\section{Izquierdas y derechas en la Argentina. Una hipótesis}

En nuestro país, la emergencia de un conglomerado de fuerzas, sectores o ideas que podrían ser definidas como de izquierda y/o de derecha siguió básicamente al itinerario político e ideológico del continente. Desenganchada tempranamente de la historia política de Europa, América Latina no adoptó la terminología parlamentaria que se desarrolló en el Viejo Continente a lo largo del s. XIX. Para el continente latinoamericano, izquierda y derecha no fueron originariamente categorías o denominaciones de los sectores políticos representados en un cuerpo legislativo. Tampoco se constituyeron en las identidades que la adhesión a esos sectores generaron entre intelectuales, periodistas y ciudadanos interesados en la política, y que recién surgieron en Europa a fines del s. XIX.

En la Argentina la izquierda como concepto político empezó por denominar al conglomerado de partidos de ideología socialista o ácrata, de carácter reformista o revolucionario, independientemente de su presencia parlamentaria. Es sabido que el socialismo argentino obtuvo tempranamente una representación en el Congreso de la Nación (1904). Pero durante mucho tiempo el socialismo se consideró a sí mismo una fuerza política en oposición al sistema político burgués, sus partidos y sus instituciones, sus formas representación y participación del poder, negándose a entrar en la contienda electoral. Lo mismo sucedió a su tiempo con los comunistas y anarquistas.

Esta ausencia de referencias parlamentarias, que definieran una identidad extensa más allá de estas organizaciones políticas referidas (tal como sucedía en Europa), hizo que fuese posible hablar de la izquierda por un lado y de otras organizaciones políticas que no reconocían posicionamientos dentro del eje izquierda-derecha. Las categorizaciones en esta línea -muy marginales, originadas en los propios intelectuales y partidos de izquierda- 
datan de mediados de los años treinta, momento en el que los partidos comunistas que operaban en los países democráticos recibieron la directiva política del Comintern de formar frentes populares con partidos burgueses con el objeto de oponerse al avance del fascismo. El objeto de la rehabilitación de la categoría izquierda -tradicionalmente muy maltratada por el marxismo leninismo- fue generar esa identidad extensa con las organizaciones políticas burguesas que permitiera la formación de coaliciones amplias.

El advenimiento del peronismo y su configuración transversal respecto del espectro político bipolar impediría durante al menos una década la formación de una identidad diversa e inclusiva de izquierda que trascendiera las fronteras de las pequeñas organizaciones socialistas y comunistas. No obstante, con el tiempo sería el propio peronismo el espacio político que se constituiría como la condición de posibilidad de un espectro bipolar horizontal. No como elemento discriminador de posiciones de las diversas fuerzas políticas del país a lo largo de un eje izquierda-derecha (como a priori podría suponerse) sino como articulación interna de tendencias que operaban en su seno.

Hemos explicado que el contexto de aparición de los conceptos políticos posrevolucionarios de izquierda y derecha fue el de los recintos asamblearios instituidos en Europa con el advenimiento de las revoluciones burguesas. En esos cuerpos deliberativos se encontraban representados, sino todos, una buena parte de las diversas formas de concebir el orden institucional y la orientación política de sus Estados. En la Argentina estos cuerpos deliberativos tuvieron una existencia precaria e intermitente, y cuando se consolidaron apenas pudieron ampliar la representación de sectores sociales y políticos a partir de las primeras décadas del s. XX.

En un contexto de contestación -en diversas formas y desde distintas tradiciones ideológicas- del sistema democrático liberal como se dio en las décadas de 1930 y 1940 es probable que no fuera precisamente una asamblea o un órgano deliberativo el lugar en el que se articulara una representación adecuada de diversos actores políticos y sociales. Quizá únicamente un movimiento, una entidad capaz de integrar no solamente fuerzas políticas sino organizaciones sociales y sindicatos, pudiera hacerlo. La Revolución Libertadora potenció una discusión en el seno del peronismo que ya venía dándose marginalmente: su propia evolución como fuerza política y como proyecto nacional.

Desde sectores fuertemente inspirados en el marxismo leninismo (vamos a dejar de lado la discusión de si lo hicieron a partir de su variante trotskista) se advirtió que hasta ese momento el peronismo había cumplido una fase inicial del proceso de liberación nacional y a partir de entonces, con el revés sufrido en septiembre de 1955, era preciso avanzar decididamente en un sentido socialista y revolucionario. Otros sectores se mantuvieron fieles a las formas, las estructuras y los principios que Perón había dado al movimiento, constituyéndose en guardianes de la ortodoxia. No habría modificaciones sustanciales al peronismo tal como lo había concebido Perón. La confrontación entre estos sectores duraría al menos dos décadas.

En 1960, Juan José Hernández Arregui (1973) designa -de forma provisional, como él mismo advierte- a esta corriente socialista y revolucionaria vinculada al peronismo como Izquierda Nacional, en contraposición a la izquierda liberal, constituida por las formaciones socialistas y comunistas, que se habían integrado a la partidocracia liberal y seguían líneas políticas que calificaba de antinacionales y burguesas (475-477). La definición de Hernández Arregui no solamente introdujo la distinción izquierda-derecha en el seno del peronismo sino que además la liberó de sus límites anteriores, su constricción a los partidos de izquierda, generando por primera vez en la Argentina una identidad extensa que llegaba hasta el corazón de la principal organización política y social del país.

Si se siguen los criterios de identificación de la distinción (alineación horizontal según grados de moderación/radicalización, simetría especular) puede afirmarse que la emergencia de lo que se conoce como la Nueva Izquierda, a partir de fines de los cincuenta y principios de los sesenta, quizá debería ser considerada como la primera izquierda argentina propiamente dicha, porque desborda lo estrictamente partidario, dándole por primera vez un sentido propio, una identidad difusa que no se vincula necesariamente con una militancia en determinadas organizaciones políticas. 
Si se atiende al otro lado del espectro del movimiento nacional justicialista, se advierte que la emergencia de una derecha peronista responde básicamente, según la dinámica que hemos expuesto antes, a una identidad heteroatribuida, que nunca termina de ser propia, pero que probablemente esté más cerca que ninguna otra de constituirse como tal.

Independientemente de grupos sociales, instituciones u organizaciones políticas identificadas con ideas de tipo conservador, liberal, tradicional-confesional o nacionalista, es decir, de la constelación de ideas y actores políticos que se sitúan a la derecha del espectro, lo más parecido a una derecha autoconsciente fueron los sectores que adhirieron a la ortodoxia del peronismo y a la subordinación indiscutida al conductor del movimiento. No es casual que precisamente en el seno del movimiento peronista se diera el surgimiento de una organización que planteara de forma explícita su condición de anti-izquierda: la Alianza Anticomunista Argentina.

Izquierda y derecha en la Argentina se articulaban dentro del organismo político y social más importante del país, el cual, en cierto sentido, parecía tener mayor potencial de representatividad de la complejidad y la variedad de las identidades nacionales que la institución liberal por excelencia: el mismísimo Congreso de la Nación.

\section{Esta compilación}

Se reúnen en el presente dossier tres estudios que exploran la emergencia de organizaciones políticas de derecha en el contexto de la proscripción del peronismo y de reorganización del movimiento.

En el primero, “Antecedentes ideológicos del primer núcleo del Movimiento Nacionalista Tacuara (1956-1958)”, se realiza una valiosa reconstrucción historiográfica de la tradición nacionalista -heterogénea y permeable- de la cual se nutrió Tacuara. Mario Jiménez estudia con entrevistas y fuentes primarias los dos primeros años del movimiento. Parte de la hipótesis de que sus "rasgos ideológicos e identitarios deben ser pensados desde la historicidad de la agrupación”, por ello su análisis del "núcleo original” de Tacuara entre 1956 y 1958 es puesto en diálogo con la trayectoria del nacionalismo.

Jiménez analiza, entre otros documentos, el "Programa básico revolucionario" aprobado en el Primer Congreso Nacional realizado en Marcos Paz. Destaca las reminiscencias con el falangismo español y las herencias del "nacionalismo restaurador". Asimismo, muestra las tensiones entre tradición y renovación, considerando la diversidad de atmósferas entre la Argentina de entreguerras y de la primera época post-peronista, las radicales transformaciones en el orden internacional de la segunda posguerra y sus repercusiones en el escenario local.

En el segundo estudio, "Bajo fuerzas de ocupación. Violencia y revolución en Trinchera de la Juventud peronista (1960-1963), se abordan los conceptos de violencia y de revolución en la revista Trinchera de la "Mesa ejecutiva de la Juventud Peronista de la Capital Federal y del Gran Buenos Aires”. La obra de Hannah Arendt forma parte del marco teórico desde el cual Andrés Funes analiza estos conceptos. La propuesta del núcleo promotor de la revista es la recuperación y, a la vez, radicalización del legado o naturaleza de la truncada revolución nacional justicialista, heredera de las banderas del primer 17 de octubre de 1945.

En el contexto de proscripción y persecución del peronismo, los militantes juveniles de la Mesa miraban su patria desde el ángulo de una profunda crisis, económica, social, política, de la que solo la revolución -necesaria, impostergable, violenta y sin negociaciones, entendida como insurrección o levantamiento popular, no conspiración militar- liberaría al pueblo y a la patria, de la opresión, la oligarquía, el imperialismo, los cipayos, en definitiva, de los “eternos enemigos". 
El regreso del General Perón constituía el objetivo de una revolución legitimada por la "fuerza de las circunstancias”, y cuyo artífice "únicamente” sería el pueblo. La juventud peronista -autodefinida como heroica, abnegada, ascética y desinteresada- se posicionaba en un lugar de vanguardia "armada”, y a la vez en actitud de guardia, de lucha frente a los retardatarios, traidores o “ajenos al espíritu revolucionario”, que pretendían retener las riendas del movimiento desde adentro. Una revolución entendida como un radical cambio de las estructuras: sobre las ruinas del "Estado liberal burgués” construir el Estado justicialista.

En el tercer artículo, "Los muchachos peronistas antijudíos: a propósito del antisemitismo en el movimiento peronista”, se estudia el rebrote del discurso antisemita en un significativo sector de la derecha peronista, a través de sus publicaciones (por ejemplo, Patria Libre, Patria Bárbara, Retorno y el semanario Huella) y otros textos en los tempranos años sesenta. En 1962, la llegada a la Argentina del tunecino Hussein Triki, representante de la Liga Árabe, activo militante contra el Estado de Israel contribuyó a reforzar el componente antisionista al larvado antisemitismo del peronismo y del nacionalismo.

El estudio del especialista en la derecha peronista Juan Luis Besoky reconoce que el peronismo también aglutinó a militantes judíos, aunque no puede soslayarse la presencia de activos antisemitas en las filas de la derecha del movimiento, inscriptos en una tradición nacionalista más antigua donde la “cuestión judía” había sido visualizada como "problema nacional”. Besoky reconstruye la funcionalidad discursiva que tuvo en la derecha peronista el concepto de sinarquía para englobar un conjunto heterogéneo de enemigos conspirativos globales: judíos, masones, sionistas, liberales, comunistas, jesuitas, "grandes internacionales”, enemigos que se "infiltraban” en el movimiento.

\section{Notas}

1 El presente dossier ha sido recopilado en el marco del proyecto de investigación El uso y las inflexiones de los conceptos de izquierda y derecha en la cultura política argentina. Aproximaciones desde la historia de las ideas y los conceptos. Segunda parte (G032), otorgado por la Secretaría de Ciencia, Técnica y Posgrado de la Universidad Nacional Cuyo.

$\underline{2}$ Esto quiere decir que debe existir una contrariedad de valores, ideales, principios o líneas de acción política entre derecha e izquierda. A un elemento presente en la izquierda debe corresponder otro opuesto o contrario en la derecha. Es el caso de la definición de izquierda y derecha que propone Norberto Bobbio (1995). Para el célebre politólogo italiano, la izquierda se identifica con la igualdad y la derecha con la desigualdad. Una crítica de las tesis de Bobbio puede verse en Ghiretti (2002).

3 Véase: (Siegfried, 1930) y (Mounier, 1973).

$\underline{4}$ “Lorsqu'on me demande si la coupure entre partis de droite et de gauche, entre hommes de droite et hommes de gauche a encore un sens, la première idée qui me vient est que l'homme qui me pose cette question n'est certainement pas un homme de la gauche”. La reflexión de Alain ha sido profusamente empleada por los autores de izquierda para definir la actitud genérica de la derecha hacia la distinción. Véase, por ejemplo, Aron (1971: 9); Sassoon (1996: 776); Giddens (1998: 51) Cotarelo (1989: 15); Haro Tecglen (1995: 174); Hope (1966: 99); Campi (1997: 153, 157); Gómez Marín (1996: 37) y Gauchet (1992: 426); Comte Sponville (2003: 146). También aparece en autores que no pertenecen a la izquierda. Es el caso de Molina Cano (1999: 55-67); De Benoist (1997:

77) y Tenzer (1992: 234). Sin embargo, un repaso a las recientes publicaciones, foros de debate y discusiones públicas sobre el asunto revelarían, como ha puesto de manifiesto el mismo Bobbio, una clara predominancia de autores de izquierda. Por detrás de estas reacciones puede escucharse el eco de las palabras de Carl Schmitt: "quien se encuentra en lucha con un enemigo absoluto -trátese de un enemigo de clase o de raza o de un enemigo eterno sin límites- no está interesado en nuestras preocupaciones relativas a lo "político”; por el contrario, ve en 
ello una amenaza a su capacidad inmediata de lucha, un debilitamiento suyo a través de la reflexión, una hamletización y una relativización sospechosa, del mismo modo que Lenin rechaza el 'objetivismo' de Struve”. Schmitt (1985: 24).

\section{Referencias bibliográficas}

Alain (seud. de Émile Chartier, 1934). Propos de politique. Paris: Rieder.

Aron, R. (1955). L'opium des intellectuels. Paris: Calmann-Levy.

Bobbio, N. (1995). Destra e sinistra. Ragioni e significati di una distinzione política. 2ed. Roma: Donzelli.

Bueno, G. (2003). El mito de la izquierda. Barcelona: Ediciones B.

Campi, A. (1997). "La dicotomia destra-sinistra: ragioni di una crisi”, en Campi, A. y Santambrogio, A. (eds.). Destra/Sinistra. Storia e fenomenologia di una dicotomia politica. Roma: Antonio Pellicani.

Campi, A. y Santambrogio, A. (eds.) (1997). Destra/Sinistra. Storia e fenomenologia di una dicotomia politica. Roma: Antonio Pellicani.

Comte Sponville, A. (2003). Diccionario filosófico. Barcelona: Paidós.

Cotarelo, R. (1989). La izquierda: desengaño, resignación y utopía. Barcelona: Ediciones del Drac.

De Benoist, A. (1997). "La fine della dicotomia Destra/Sinistra”, en Campi, A. y Santambrogio, A. (eds.). Destra/Sinistra. Storia e fenomenologia di una dicotomia politica. Roma: Antonio Pellicani.

Duclerc, E. y Pagnerre (1848). Dictionnaire politique. Paris: Pagnerre.

Gauchet, M. (1992). “La droite et la gauche”, en Nora, P. (dir.). Les lieux de mémoire. III. Les France. Conflits et partages. Paris: Gallimard.

Ghiretti, H. (2002). La izquierda. Usos, abusos, precisiones y confusiones. Barcelona: Ariel, 2002.

Giddens, A. (1998). La tercera vía. La renovación de la socialdemocracia. Madrid: Taurus.

Gómez Marín, J. A. (1996). Hablar con propiedad. Antología de frases de derecha. Barcelona: Libertarias/Prodhufi.

Haro Tecglen, Eduardo (1995). Diccionario político. Barcelona: Planeta.

Hernández Arregui, J. (1973). La formación de la conciencia nacional. 3ed. Buenos Aires: Plus Ultra.

Hope, F. (1966). “The Intellectual Left”, en Kaufman, G. The Left. A symposium. London: Anthony Blond.

Lanzmann, C. (1971). El hombre de izquierda. Buenos Aires: La pléyade.

Madiran, J. (1979). La izquierda y la derecha. Buenos Aires: Iction.

Molina Cano, J. (1999). “El liberalismo y la querella impolítica de la izquierda y la derecha”, Veintiuno. Revista de pensamiento y cultura, (Madrid, Fundación Cánovas del Castillo), nº43, 55-67.

Mounier, E. (1973). “Breve tratado sobre la mítica de izquierda” en Comunismo, anarquía, personalismo. Bilbao: Zero [1938].

Sassoon, D. (1996). One Hundred Years of Socialism. The Western European Left in the Twentieth Century. New York: The New Press. 
Schmitt, C. (1985). El concepto de lo “político”. Teoría del partisano. Notas complementarias al concepto de lo “político". México: Folios.

Siegfried, A. (1930). Tableau des partis en France. Paris: Bernard Grasset.

Tenzer, N. (1992). La sociedad despolitizada. Ensayo sobre los fundamentos de la política. Barcelona: Paidós.

Voegelin, E. (1968). Nueva ciencia de la política. Madrid: Rialp. 